\title{
Integrals in Left Coideal Subalgebras and Group-Like Projections
}

\author{
Alexandru Chirvasitu ${ }^{1} \cdot$ Paweł Kasprzak $^{2}$ (D) $\cdot$ Piotr Szulim $^{3}$
}

Received: 19 October 2018 / Accepted: 3 April 2019 / Published online: 25 May 2019

(C) The Author(s) 2019

\begin{abstract}
We develop a theory of right group-like projections in Hopf algebras linking them with the theory of left coideal subalgebras with two sided counital integrals. Every right group-like projection is associated with a left coideal subalgebra, maximal among the ones containing the given group-like projection as an integral, and we show that that subalgebra is finite dimensional. We observe that in a semisimple Hopf algebra $H$ every left coideal subalgebra has an integral and we prove a 1-1 correspondence between right group-like projections and left coideal subalgebras of $H$. We provide a number of equivalent conditions for a right group-like projections to be left group-like projection and prove a 1-1 correspondence between semisimple left coideal subalgebras preserved by the squared antipode and two sided group-like projections. We also classify left coideal subalgebras in Taft Hopf algebras $H_{n^{2}}$ over a field $\mathbb{k}$, showing that the automorphism group splits them into

- a class of cardinality $|\mathbb{k}|-1$ of semisimple ones which correspond to right group-like projections which are not two sided;

- finitely many semisimple singletons, each corresponding to two sided group-like projection; the number of those singletons for $H_{n^{2}}$ is equal to the number of divisors of $n$;

- finitely many singletons, each non-semisimple and admitting no right group-like projection; the number of those singletons for $H_{n^{2}}$ is equal to the number of divisors of $n$;
\end{abstract}

In particular we answer the question of Landstad and Van Daele showing that there do exist right group-like projections which are not left group-like projections.

Keywords Group-like projection · Left coideal subalgebra · Taft Hopf algebra

Presented by: Vyjayanthi Chari

Paweł Kasprzak

pawel.kasprzak@fuw.edu.pl

Extended author information available on the last page of the article. 


\section{Introduction}

In order to explain the classical motivation standing behind the concept of group like projection let us fix a finite group $G$ and consider Hopf algebra of complex functions $\mathrm{C}(G)$ on $G$. It is easy to check that given a subgroup $H \subset G$, its indicator function $\mathbb{1}_{H} \in \mathrm{C}(G)$ is a projection satisfying

$$
\Delta\left(\mathbb{1}_{H}\right)\left(\mathbb{1}_{G} \otimes \mathbb{1}_{H}\right)=\mathbb{1}_{H} \otimes \mathbb{1}_{H} .
$$

Conversely, a non-zero projection $P \in \mathrm{C}(G)$ satisfying $\Delta(P)(\mathbb{1} \otimes P)=P \otimes P$ is of the form $P=\mathbb{1}_{H}$. In particular $\Delta(P)(\mathbb{1} \otimes P)=P \otimes P$ if and only if $P=\mathbb{1}_{H}$ if and only if $\Delta(P)(P \otimes \mathbb{1})=P \otimes P$. It turns out that there are Hopf algebras admitting an element $P$ satisfying

$$
\Delta(P)(\mathbb{1} \otimes P)=P \otimes P=(\mathbb{1} \otimes P) \Delta(P)
$$

but not satisfying

$$
\Delta(P)(P \otimes \mathbb{1})=P \otimes P=(P \otimes \mathbb{1}) \Delta(P),
$$

thus we use the terminology of right and left group-like projections for the elements satisfying the former or the latter equation respectively.

A right group-like projection $P$ in a Hopf algebra $\mathcal{A}$ cannot, in general, be matched with a quotient Hopf subalgebra, thus we do not have a full analogy with the classical case. Remarkably however, $P$ can be matched with a left coideal subalgebra denoted by $\mathcal{N}_{P} \subset \mathcal{A}$, which is the analog of the algebra of functions on the quotient space $G / H$ from the classical context.

$\mathcal{N}_{P}$ is characterized as being a maximal among all left coideal subalgebras $\mathcal{N} \subset \mathcal{A}$ containing $P$ and such that $P x=x P=\varepsilon(x) P$ for all $x \in \mathcal{N}$. Denoting $\mathcal{V}_{P}=$ $\{(\mu \otimes \mathrm{id})(\Delta(P)): \mu \in \mathcal{A}\}$ we clearly have $\mathcal{V}_{P} \subset \mathcal{N}_{P}$. Having proved that $\mathcal{N}_{P}$ is finite dimensional (c.f. Corollary 3.15) allowed us to use [8] to conclude that $\mathcal{N}_{P}=\mathcal{V}_{P}$ if and only if $\mathcal{N}_{P}$ is quasi-Frobenius, Theorem 3.17. In particular this holds in the following three cases

- $\quad P$ is two sided group-like projection, Proposition 3.21;

- $\quad \mathcal{N}_{P}$ is semisimple, Proposition 3.23;

- $\mathcal{A}$ is weakly finite, Corollary 3.20.

We also prove that that $\mathcal{N}_{P}=\mathcal{V}_{P}$ if and only if $\mathbb{1}_{\mathcal{A}} \in \mathcal{V}_{P}$

The semisimplicity is the property which turns out to link nicely with the theory of group-like projections in the following two fundamental ways:

- $\quad$ if $\mathcal{A}$ is a semisimple Hopf algebra then we have a 1-1 correspondence between left coideal subalgebras of $\mathcal{A}$ and right group-like projections in $\mathcal{A}$, see Theorem 3.35;

- $\quad P \mapsto \mathcal{N}_{P}$ restricts to a one two one correspondence between two sided group-like projections in $\mathcal{A}$ and semisimple left coideals subalgebras $\mathcal{N} \subset \mathcal{A}$ preserved by the square of the antipode, see Theorem 3.24.

Using [8] we also provide a necessary and sufficient condition for $\mathcal{N}_{P}$ to be semisimple, see Theorem 3.22. The results described above are contained in Section 3.

In Section 4 we classify left coideals subalgebras in Taft Hopf algebras $H_{n^{2}}$ over a field $\mathbb{k}$, where $n \in \mathbb{N}$. It turns our that the automorphism group of $H_{n^{2}}$ splits them into

- a class of cardinality $|\mathbb{k}|-1$ of semisimple ones which correspond to right group-like projections which are not two sided; 
- a finite number of semisimple singletons each corresponding to two sided group-like projection; the number of those singletons for $H_{n^{2}}$ is equal to the number of divisors of $n$;

- finite number of singletons each being non-semisimple and not admitting a right grouplike projection; the number of those singletons for $H_{n^{2}}$ is equal to the number of divisors of $n$.

Every coideal and every group-like projection has an explicit description. Our classification enabled us to answer the question of Landstad and Van Daele showing that there do exist right group-like projections which are not left group-like projections (already in 4-dimensional Taft algebra $\mathrm{H}_{4}$ ).

In Section 2 we define left, right and two sided group-like projections together with their shifts and we prove a few preliminary results.

For background on Hopf algebras we refer to $[13,16]$. We shall freely use the concept of semisimple and cosemisimple Hopf algebra, coradical filtration, primitive elements etc. It is assumed that the reader is familiar with the basics of the theory of modules over rings, see e.g. [9]. We shall make occasional use of such concepts as injective modules, faithfully flat algebras over subalgebras, quasi-Frobenius algebras and weakly finite algebras.

The study initiated in this paper is continued in [6] where the theory of generalized integrals and cointegrals on a left coideal subalgebra is developed. In particular the finite dimensionality result of Corollary 3.15 holds also in the case when a given left coideal subalgebra $\mathcal{N} \subset \mathcal{A}$ equipped with a non-zero multiplicative functional $\mu \in \mathcal{N}$ admits a non-zero $\Lambda \in \mathcal{N}$ satisfying $a \Lambda=\mu(a) \Lambda$ for all $a \in \mathcal{N}$, [6, Theorem 3.7.]. The issue of existence of such $\Lambda \in \mathcal{N}$ turns out to be a non-trivial matter, linked e.g. with the existence of generalized cointegrals on $\mathcal{N}$.

\section{Preliminaries}

We shall assume that $(\mathcal{A}, \Delta, S, \varepsilon)$ is a Hopf algebra (over an arbitrary field $\mathbb{k}$ ) with an invertible antipode. We shall often write that $\mathcal{A}$ is a Hopf algebra, having in mind that comultiplication $\Delta$, coinverse $S$ and counit $\varepsilon$ are fixed. We shall use the Sweedler's notation: $\Delta(x)=x_{(1)} \otimes x_{(2)}$. A vector subspace $\mathcal{V} \subset \mathcal{A}$ is said to be a left coideal if $\Delta(\mathcal{V}) \subset \mathcal{A} \otimes \mathcal{V}$. A unital subalgebra $\mathcal{N} \subset \mathcal{A}$ which is a left coideal is said to be a left coideal subalgebra. A Hopf surjection $\pi: \mathcal{A} \rightarrow \mathcal{B}$ can be assigned with a left coideal subalgebra $\mathcal{N}_{\pi}=\left\{x \in \mathcal{A}:(\mathrm{id} \otimes \pi)(\Delta(x))=x \otimes \mathbb{1}_{\mathcal{B}}\right\}$. It is easily seen that $\mathcal{N}_{\pi}$ is preserved by the adjoint action $\operatorname{Ad}: \mathcal{A} \otimes \mathcal{A} \rightarrow \mathcal{A}$ of $\mathcal{A}$ on itself, where $\operatorname{Ad}(a \otimes x)=a_{(1)} x S\left(a_{(2)}\right)$. A left coideal subalgebra $\mathcal{N}$ is said to be normal if the adjoint action restricts to $\mathcal{A} \otimes \mathcal{N} \ni a \otimes x \mapsto a_{(1)} x S\left(a_{(2)}\right) \in \mathcal{N}$. It is well known that when $\mathcal{A}$ is left faithfully flat over a normal left coideal subalgebra then $\mathcal{N}=\mathcal{N}_{\pi}$ for a faithfully coflat Hopf projection $\pi$, unique in the sense that its kernel is uniquely determined by $\mathcal{N}$.

Let $\left(\mathcal{C}, \Delta_{\mathcal{C}}, \varepsilon\right)$ be a coalgebra and let $\pi: \mathcal{A} \rightarrow \mathcal{C}$ be surjective map of coalgebras. If in addition $\mathcal{C}$ is equipped with a left $\mathcal{A}$-module structure $\mathcal{A} \otimes \mathcal{C} \rightarrow \mathcal{C}$ which is a coalgebra map and $\pi: \mathcal{A} \rightarrow \mathcal{C}$ is $\mathcal{A}$ linear then we say that $\mathcal{C}$ is a left module coalgebra quotient. Given a left module coalgebra quotient $\pi: \mathcal{A} \rightarrow \mathcal{C}$ we assigned to it a left coideal subalgebra of $\mathcal{A}$

$$
\mathcal{N}_{\pi}=\{x \in \mathcal{A}:(\mathrm{id} \otimes \pi)(\Delta(x))=x \otimes \pi(\mathbb{1})\} .
$$


Conversely given a left coideal subalgebra $\mathcal{N}$ we assign to it a left module coalgebra quotient $\pi: \mathcal{A} \rightarrow \mathcal{C}_{\mathcal{N}}$ where $\mathcal{C}_{\mathcal{N}}=\mathcal{A} /\left(\mathcal{A N}{ }^{-}\right)$and $\mathcal{N}^{-}=\mathcal{N} \cap \operatorname{ker} \varepsilon$. If $\mathcal{A}$ is left $\mathcal{N}$-faithfully flat then $\mathcal{N}_{\mathcal{C}_{\mathcal{N}}}=\mathcal{N}$. Let us move on to the main subject of this paper.

Definition 2.1 Let $\mathcal{A}$ be a Hopf algebra and $P \in \mathcal{A}$ a non-zero projection. We say that

(1) $P$ is a right group-like projection if

$$
\Delta(P)(\mathbb{1} \otimes P)=P \otimes P=(\mathbb{1} \otimes P) \Delta(P)
$$

(2) $P$ is a left group-like projection if

$$
\Delta(P)(P \otimes \mathbb{1})=P \otimes P=(P \otimes \mathbb{1}) \Delta(P)
$$

(3) $P$ is a two sided group-like projection (or simply a group-like projection) if it is a left and right group-like projection.

In the course of the paper we shall use the following operators on $\mathcal{A} \otimes \mathcal{A}$ (the formulas below are extended to an arbitrary element of $\mathcal{A} \otimes \mathcal{A}$ be linearity):

$$
\begin{aligned}
\mathrm{W}_{l r}(x \otimes y) & =x_{(1)} \otimes x_{(2)} y, \\
\mathrm{~W}_{l l}(x \otimes y) & =x_{(1)} \otimes y x_{(2)}, \\
\mathrm{W}_{r l}(x \otimes y) & =x y_{(1)} \otimes y_{(2)}, \\
\mathrm{W}_{r r}(x \otimes y) & =y_{(1)} x \otimes y_{(2)} .
\end{aligned}
$$

In our notation $\mathrm{W}_{-,-}$the first index establishes the position of the tensorand to which the comultiplication is applied whereas the second index corresponds to the position of the remaining tensorand in the multiplication. The operators $\mathrm{W}_{-,-}$are invertible and we have

$$
\begin{aligned}
& \mathrm{W}_{l r}^{-1}(x \otimes y)=x_{(1)} \otimes S\left(x_{(2)}\right) y \\
& \mathrm{~W}_{l l}^{-1}(x \otimes y)=x_{(1)} \otimes y S^{-1}\left(x_{(2)}\right) \\
& \mathrm{W}_{r l}^{-1}(x \otimes y)=x S\left(y_{(1)}\right) \otimes y_{(2)} \\
& \mathrm{W}_{r r}^{-1}(x \otimes y)=S^{-1}\left(y_{(1)}\right) x \otimes y_{(2)} .
\end{aligned}
$$

Note that $P \in \mathcal{A}$ is a right group-like projection if and only if

$$
\mathrm{W}_{l r}(P \otimes P)=P \otimes P=\mathrm{W}_{l l}(P \otimes P) .
$$

Similarly $P$ is a left group-like projection if and only if

$$
\mathrm{W}_{r r}(P \otimes P)=P \otimes P=\mathrm{W}_{r l}(P \otimes P) .
$$

As mentioned in the introduction, if $G$ is a finite group and $P \in \mathrm{C}(G)$ is a group like projection then $P=\mathbb{1}_{H}$ where $H \subset G$ is a subgroup of $G$. The projection $Q$ entering the next lemma is the analog of $\mathbb{1}_{H g}$ for some $g \in G$.

Lemma 2.2 Let $y \in \mathcal{A}$ and $Q \in \mathcal{A}$ be a non-zero projection such that

$$
(\mathbb{1} \otimes Q) \Delta(Q)=\Delta(Q)(\mathbb{1} \otimes Q)=y \otimes Q .
$$

Then $y$ is a right group-like projection. Moreover if $S^{2}(Q)=Q$ then $S^{2}(y)=y$.

Proof The identity

$$
(\mathbb{1} \otimes y) \Delta(y)=y \otimes y
$$


is the consequence of the following computation

$$
\begin{aligned}
((\mathbb{1} \otimes y) \Delta(y)) \otimes Q & =(\mathbb{1} \otimes y \otimes \mathbb{1})(\Delta \otimes \mathrm{id})(y \otimes Q) \\
& =(\mathbb{1} \otimes y \otimes Q)(\Delta \otimes \mathrm{id})(\Delta(Q)) \\
& =(\mathbb{1} \otimes \mathbb{1} \otimes Q)(\mathbb{1} \otimes \Delta(Q))(\mathrm{id} \otimes \Delta)(\Delta(Q)) \\
& =(\mathbb{1} \otimes \mathbb{1} \otimes Q)(\mathrm{id} \otimes \Delta)((\mathbb{1} \otimes Q) \Delta(Q)) \\
& =(\mathbb{1} \otimes \mathbb{1} \otimes Q)(\mathrm{id} \otimes \Delta)(y \otimes Q) \\
& =(y \otimes y) \otimes Q .
\end{aligned}
$$

Similarly we check that $\Delta(y)(\mathbb{1} \otimes y)=y \otimes y$. Since $((\mathbb{1} \otimes Q) \Delta(Q))^{2}=(\mathbb{1} \otimes Q) \Delta(Q)$ we see that $(Q \otimes y)$ and thus $y$ are projections.

Remark 2.3 There is a left version of Lemma 2.2 with $Q$ and $y \in \mathcal{A}$ satisfying $\Delta(Q)(Q \otimes$ $\mathbb{1})=(Q \otimes \mathbb{1}) \Delta(Q)=Q \otimes y$ and $y$ being a left group-like projection. In particular if $P \in \mathcal{A}$ is a right group-like projection admitting a non-zero $Q \in \mathcal{A}$ satisfying $\Delta(Q)(Q \otimes \mathbb{1})=$ $Q \otimes P=(Q \otimes \mathbb{1}) \Delta(Q)$ then $P$ is a two sided group-like projection.

Definition 2.4 Let $P \in \mathcal{A}$ be a right group-like projection. We say that a projection $Q \in \mathcal{A}$ is a right shift of $P$ if

$$
\Delta(Q)(\mathbb{1} \otimes Q)=P \otimes Q=(\mathbb{1} \otimes Q) \Delta(Q) .
$$

Let $P$ be a left group-like projection. We say that a projection $Q \in \mathcal{A}$ is a left shift of $P$ if

$$
\Delta(Q)(Q \otimes \mathbb{1})=Q \otimes P=(Q \otimes \mathbb{1}) \Delta(Q) .
$$

Lemma 2.5 Let $P, Q \in \mathcal{A}$ be non-zero projections. The following conditions are equivalent

- $Q$ is a right shift of a right group-like projection $P$;

- $P$ and $Q$ satisfy $\left(\mathbb{1} \otimes S^{-1}(Q)\right) \Delta(P)=Q \otimes S^{-1}(Q)$ and $\Delta(P)(\mathbb{1} \otimes S(Q))=Q \otimes S(Q)$.

In particular if $Q$ is a right shift of $P$ then there exists a functional $\mu \in \mathcal{A}^{*}$ such that $Q=(\mathrm{id} \otimes \mu)(\Delta(P))$. Moreover $S^{2}(Q)=Q$ if and only if $S^{2}(P)=P$.

The condition $S^{2}(P)=P$ for a right group-like projection $P$ turns out to be equivalent with $P$ being a two sided group-like projection, c.f. Theorem 3.5.

Proof of Lemma 2.5 In order to get the equivalence of the first and the second bullet point it is enough to write (2.5) in the form

$$
\mathrm{W}_{l r}(Q \otimes Q)=P \otimes Q=\mathrm{W}_{l l}(Q \otimes Q)
$$

and use the inverse formulas (2.1). The existence of $\mu \in \mathcal{A}^{*}$ satisfying $(\operatorname{id} \otimes \mu)(\Delta(P))=Q$ is obvious.

If $S^{2}(Q)=Q$ then $\left(S^{2} \otimes S^{2}\right)(\Delta(Q)(\mathbb{1} \otimes Q))=\Delta(Q)(\mathbb{1} \otimes Q)$ which implies that $S^{2}(P) \otimes Q=P \otimes Q$ and thus $S^{2}(P)=P$. For the converse implication suppose that $S^{2}(P)=P$. Applying $S^{2} \otimes S^{2}$ to the equality

$$
Q \otimes S^{-1}(Q)=\left(\mathbb{1} \otimes S^{-1}(Q)\right) \Delta(P)\left(\mathbb{1} \otimes S^{-1}(Q)\right)
$$

we get $S^{2}(Q) \otimes S(Q)=(\mathbb{1} \otimes S(Q)) \Delta(P)(\mathbb{1} \otimes S(Q))=Q \otimes S(Q)$ and we conclude that $S^{2}(Q)=Q$. 


\section{General Results}

In the first part of this section we shall derive a number of equivalent conditions for a right group-like projection to be two sided, c.f. Section 3.1. In the second part Section 3.2 we shall relate the theory of (right) group-like projections with the theory of left coideal subalgebras with integrals developed in [8]. The third subsection Section 3.3 is concerned with the normality, faithful flatness and other properties related with left coideals subalgebras assigned to right group-like projections. Finally in the fourth subsection Section 3.4 we discuss right group-like projections in a semisimple Hopf algebra $\mathcal{A}$.

\subsection{Two Sided Group-Like Projection}

Let us begin with the following auxiliary lemma.

Lemma 3.1 Let $\mathcal{A}$ be a Hopf algebra and $a, b \in \mathcal{A}$. The following two conditions are equivalent

- $\Delta(a)(\mathbb{1} \otimes b)=\Delta(b)(a \otimes \mathbb{1})$

- $\quad(b \otimes \mathbb{1})(\mathrm{id} \otimes S)(\Delta(a))=a \otimes b$

Moreover if one of these equivalent conditions holds then we have $a b=\varepsilon(a) b=S(a) \varepsilon(b)$.

Proof Suppose that $\Delta(a)(\mathbb{1} \otimes b)=\Delta(b)(a \otimes \mathbb{1})$ holds. Applying $(\varepsilon \otimes$ id) to the identity

$$
a_{(1)} \otimes a_{(2)} b=b_{(1)} a \otimes b_{(2)}
$$

we get $a b=\varepsilon(a) b$. Moreover we have

$$
\begin{aligned}
\varepsilon(a) b & =S\left(a_{(1)}\right) a_{(2)} b \\
& =S(a) S\left(b_{(1)}\right) b_{(2)}=S(a) \varepsilon(b)
\end{aligned}
$$

where the second equality was obtained by applying $m \circ(S \otimes \mathrm{id})$ to the identity $a_{(1)} \otimes a_{(2)} b=$ $b_{(1)} a \otimes b_{(2)}$.

In order to see the equivalence of the first and the second bullet point we can write the first as $\mathrm{W}_{l r}^{-1} \mathrm{~W}_{r r}(a \otimes b)=a \otimes b$ and note that $\mathrm{W}_{l r}^{-1} \mathrm{~W}_{r r}(x \otimes y)=y x_{(1)} \otimes S\left(x_{(2)}\right)$.

Corollary 3.2 Let $P \in \mathcal{A}$ be a non-zero projection such that

$$
\Delta(P)(\mathbb{1} \otimes P)=\Delta(P)(P \otimes \mathbb{1})
$$

Then $\varepsilon(P)=\mathbb{1}, S(P)=P$ and $(P \otimes \mathbb{1}) \Delta(P)=\Delta(P)(P \otimes \mathbb{1})=P \otimes P$. In particular

- $\quad$ every projection $P$ satisfying (3.1) is a two sided group-like projection;

- $\quad$ every two sided group-like projection $P$ satisfies $S(P)=P$.

Proof Putting $a=b=P$ in Lemma 3.1 we get $P^{2}=\varepsilon(P) P$ thus $\varepsilon(P)=1$ and $S(P)=$ $\varepsilon(P) S(P)=P$. This together with $(P \otimes \mathbb{1})(\mathrm{id} \otimes S)(\Delta(P))=P \otimes P$ (c.f. the second bullet point of Lemma 3.1) yields $(P \otimes \mathbb{1}) \Delta(P)=P \otimes P$. Applying $S \otimes S$ to the last identity we get $P \otimes P=\Delta(P)(\mathbb{1} \otimes P)=\Delta(P)(P \otimes \mathbb{1})$.

It turns out the condition $S^{2}(P)=P$ implies $S(P)=P$ if $P$ is a right group-like projection. More generally the following holds. 
Lemma 3.3 Let $P \in \mathcal{A}$ be a non-zero projection such that

$$
\Delta(P)(\mathbb{1} \otimes P)=P \otimes P
$$

If $S^{2}(P)=P$ then $S(P)=P$.

Proof Applying ( $\varepsilon \otimes$ id) to Eq. 3.2 we get $\varepsilon(P)=1$. Next, applying $m \circ(S \otimes$ id $)$ to Eq. 3.2 we get $P=S(P) P$. Thus $S(P)=S(P) S^{2}(P)=S(P) P=P$.

Let us give one more condition guaranteeing a right group-like projection to be preserved by $S$.

Lemma 3.4 Let $P \in \mathcal{A}$ be a right group-like projection. Then the following equivalence holds:

$$
\Delta(P)(\mathbb{1} \otimes P)=\Delta(P)(P \otimes \mathbb{1}) \Leftrightarrow S(P)=P
$$

Proof Clearly if $\Delta(P)(\mathbb{1} \otimes P)=\Delta(P)(P \otimes \mathbb{1})$ then $S(P)=P$ (see Corollary 3.2). Conversely, if $P$ is a right group-like projection such that $S(P)=P$ then

$$
P \otimes P=(S \otimes S)((\mathbb{1} \otimes P) \Delta(P))=\Delta^{\mathrm{op}}(P)(\mathbb{1} \otimes P)
$$

and we see that $\Delta^{\mathrm{op}}(P)(\mathbb{1} \otimes P)=\Delta(P)(\mathbb{1} \otimes P)$. Applying the flip $\sigma$, where $\sigma(a \otimes b)=$ $b \otimes a$, to both sides and using $\sigma(\Delta(P)(\mathbb{1} \otimes P))=\Delta(P)(\mathbb{1} \otimes P)$ we get $\Delta(P)(\mathbb{1} \otimes P)=$ $\Delta(P)(P \otimes \mathbb{1})$.

Note that if $P$ is a right group-like projection then $P=S(P) P$ and $S(P)=P S(P)$ (see the proof of Lemma 3.3). This observation together with other results of this subsection yields:

Theorem 3.5 Let $P \in \mathcal{A}$ be a non-zero projection. The following conditions are equivalent:

- $\quad P$ is a right group-like projection such that $S(P)=P$;

- $\quad P$ is a right group-like projection such that $S^{2}(P)=P$;

- $\quad P$ is a right group-like projection such that $P S(P)=S(P) P$;

- $\quad P$ is a left group-like projection such that $S(P)=P$;

- $\quad P$ is a left group-like projection such that $S^{2}(P)=P$;

- $\quad P$ is a left group-like projection such that $P S(P)=S(P) P$;

- $P$ is a two sided group-like projection;

- $\quad P$ satisfies $\Delta(P)(\mathbb{1} \otimes P)=\Delta(P)(P \otimes \mathbb{1})$.

Using Theorem 3.5 we can characterize two sided group-like projections it terms of their shifts, c.f. the first part of Proposition 3.6. The second part of this proposition corresponds to the classically trivial observation, that a right shift $\mathbb{1}_{H g}$ of of (two sided) group-like projection $\mathbb{1}_{H}$ is a left shift of (two sided) group-like projection $\mathbb{1}_{g^{-1}} \mathrm{Hg}$. Except generalizing this fact, Proposition 3.6 will also be essential in the proof of Proposition 3.28.

Proposition 3.6 Let $P$ be a right group like projection and let $Q$ be a right shift of $P$ in the sense of Definition 2.4. Then $P$ is a two sided group-like projection if and only if $S^{2}(Q)=Q$. In the latter case there exists a two sided group-like projection $\tilde{P}$ such that $Q$ is a left shift of $\tilde{P}$. 
Proof of Proposition 3.6 Note that the first part of the theorem follows immediately from Theorem 3.5 and Lemma 2.5 so let us move on to the proof of the second part. Using the identities entering the second bullet point of Lemma 2.5 we can check that

$$
Q S\left(P_{(2)}\right), S\left(P_{(2)}\right) Q \in \mathbb{k} Q \text {. }
$$

Applying $S \otimes S$ to these identities and using $S(P)=P$ and $S^{2}(Q)=Q$ we get

$$
Q \otimes Q=Q P_{(1)} \otimes S\left(P_{(2)}\right)=P_{(1)} Q \otimes S\left(P_{(2)}\right)
$$

which implies that

$$
Q P_{(1)} \in \mathbb{k} Q, P_{(1)} Q \in \mathbb{k} Q \text {. }
$$

Applying $\mathrm{W}_{r r}$ to the first equality in Eq. 3.4 we get $Q_{(1)} Q \otimes Q_{(2)}=S\left(P_{(3)}\right) Q P_{(1)} \otimes P_{(2)}$. Using Eqs. 3.3 and 3.5 we conclude that there exists $y$ such that

$$
\Delta(Q)(Q \otimes \mathbb{1})=Q \otimes y .
$$

Similarly using the second equality of Eq. 3.4 we get the existence of $\tilde{y}$ such that

$$
(Q \otimes \mathbb{1}) \Delta(Q)=Q \otimes \tilde{y} .
$$

Noting that $(Q \otimes \mathbb{1}) \Delta(Q)(Q \otimes \mathbb{1})=Q \otimes y=Q \otimes \tilde{y}$ we get $y=\tilde{y}$. Using Remark 2.3 we see that $y$ is a two sided group-like projection.

\subsection{Left Coideal Subalgebras with Integrals and Right Group-Like Projections}

Definition 3.7 Let $P \in \mathcal{A}$ be a right group-like projection. Consider

$$
\begin{aligned}
& \mathcal{N}_{P}^{l}=\{x \in \mathcal{A}:(\mathbb{1} \otimes P) \Delta(x)=x \otimes P\} \\
& \mathcal{N}_{P}^{r}=\{x \in \mathcal{A}: \Delta(x)(\mathbb{1} \otimes P)=x \otimes P\} \\
& \mathcal{N}_{P}=\{x \in \mathcal{A}: \Delta(x)(\mathbb{1} \otimes P)=(\mathbb{1} \otimes P) \Delta(x)=x \otimes P\}
\end{aligned}
$$

Note that these are all left coideal subalgebras and $P \in \mathcal{N}_{P}=\mathcal{N}_{P}^{l} \cap \mathcal{N}_{P}^{r}$. When we wish to refer to all three left coideal subalgebras simultaneously we write $\mathcal{N}_{P}^{-l r}$ (meaning the superscript can be blank, ' $l$ ' or ' $r$ '); similarly, ' $\mathcal{N}_{P}^{l r}$ ' means ' $\mathcal{N}_{P}^{l}$ or $\mathcal{N}_{P}^{r}$ '.

The smallest left coideal in $\mathcal{A}$ containing $P$ will be denoted $\mathcal{V}_{P}$

$$
\mathcal{V}_{P}=\left\{(\mu \otimes \mathrm{id})(\Delta(P)): \mu \in \mathcal{A}^{*}\right\} .
$$

With this description, $\mathcal{V}_{P}$ does not immediately seem to form an algebra; this issue will be discussed later.

Our first observation concerning $\mathcal{N}_{P}$ is as follows.

Proposition 3.8 Let $P \in \mathcal{A}$ be a right group-like projection and $\mathcal{N}_{P} \subset \mathcal{A}$ the associated left coideal subalgebra, as in Eq. 3.8.

Then, $P$ is a minimal central projection in $\mathcal{N}_{P}$ and $\operatorname{ker}\left(\left.\varepsilon\right|_{\mathcal{N}_{P}}\right)=(1-P) \mathcal{N}_{P}$. In particular if $\mathcal{N}_{P_{1}}=\mathcal{N}_{P_{2}}$ then $P_{1}=P_{2}$.

Proof Applying $\varepsilon \otimes$ id to the two equalities

$$
\Delta(x)(\mathbb{1} \otimes P)=x \otimes P=(\mathbb{1} \otimes P) \Delta(x), x \in \mathcal{N}_{P}
$$

shows that for $x \in \mathcal{N}_{P}$ we have $x P=\varepsilon(x) P=P x$. In particular

$$
x P=0 \Longleftrightarrow \varepsilon(x)=0 \Longleftrightarrow P x=0 .
$$


This implies both the centrality claim and the description of $\operatorname{ker}\left(\left.\varepsilon\right|_{\mathcal{N}_{P}}\right)$, while the minimality follows from this: indeed, we now know that $P \mathcal{N}_{P}$ is one-dimensional. Assuming that $\tilde{P}$ is a group-like projection such that $\mathcal{N}_{P}=\mathcal{N}_{\tilde{P}}$ we get $P=\varepsilon(P) P=P \tilde{P}=\varepsilon(\tilde{P}) \tilde{P}=$ $\tilde{P}$.

Using [18, Theorem 1] and Proposition 3.8 we get

Corollary 3.9 The number of right group like projections in a finite dimensional semisimple or cosemisimple Hopf algebra is finite.

According to Proposition 3.8 a right group-like projection $P$ has the following property:

$$
x P=\varepsilon(x) P=P x, \forall x \in \mathcal{N}_{P} .
$$

This is the special case of the condition defining two sided integrals for left or right coideals subalgebras as defined in [8, Section 2].

Definition 3.10 A left integral for a left coideal subalgebra $\mathcal{N} \subset \mathcal{A}$ is an element $\Lambda \in \mathcal{N}$ satisfying $x \Lambda=\varepsilon(x) \Lambda$ for all $x \in \mathcal{N}$. A right integral for a left coideal subalgebra $\mathcal{N} \subset \mathcal{A}$ is an element $\Lambda \in \mathcal{N}$ satisfying $\Lambda x=\varepsilon(x) \Lambda$ for all $x \in \mathcal{N}$. Finally, a two sided integral is an element that is both a left and a right integral. We say that a left, right or two sided integral $\Lambda$ is counital if $\varepsilon(\Lambda)=1$.

Proposition 3.11 Let $\mathcal{N} \subset \mathcal{A}$ be a left coideal subalgebra and $P$ be a group-like projection which is a counital two sided integral for $\mathcal{N}$. Then $\mathcal{N} \subset \mathcal{N}_{P}$. In other words $\mathcal{N}_{P}$ is a maximal left coideal subalgebra for which $P$ is a counital two sided integral.

Analogous statements hold for $\mathcal{N}_{P}^{l}\left(\mathcal{N}_{P}^{r}\right)$ and right (respectively left) integrals.

Proof We only prove the two-sided statement; the rest is entirely parallel.

It is enough to note that for all $x \in \mathcal{N}$ we have $\Delta(x) \in \mathcal{A} \otimes \mathcal{N}$ and thus $\Delta(x)(\mathbb{1} \otimes P)=$ $x \otimes P=(\mathbb{1} \otimes P) \Delta(x)$.

The theory of left coideal subalgebras with integrals developed in [8] assumes the corresponding coideals are finite dimensional. Our first aim is to show that this condition holds automatically for $\mathcal{N}_{P}$. Actually as was subsequently proved in [6] a left coideal subalgebra admitting a (generalized) non-zero integral is automatically finite dimensional. Let us first prove a technical result.

Lemma 3.12 Let $x, y \in \mathcal{A}$. The following conditions are equivalent:

- $\Delta(x)(\mathbb{1} \otimes y)=x \otimes y$;

- $\quad(\mathbb{1} \otimes x) \Delta(y)=(S(x) \otimes \mathbb{1}) \Delta(y)$.

Similarly the following conditions are equivalent:

- $\quad(\mathbb{1} \otimes y) \Delta(x)=x \otimes y$;

- $\Delta(y)(\mathbb{1} \otimes x)=\Delta(y)\left(S^{-1}(x) \otimes \mathbb{1}\right)$.

Proof Let us prove the equivalence of the first and the second bullet point of the lemma.

If $x_{(1)} \otimes x_{(2)} y=x \otimes y$ then $x_{(1)} \otimes x_{(2)} y_{(1)} \otimes x_{(3)} y_{(2)}=x \otimes y_{(1)} \otimes y_{(2)}$ which implies that $y_{(1)} \otimes x y_{(2)}=S(x) y_{(1)} \otimes y_{(2)}$. 
Conversely, if $y_{(1)} \otimes x y_{(2)}=S(x) y_{(1)} \otimes y_{(2)}$ then $y_{(1)} \otimes x_{(1)} y_{(2)} \otimes x_{(2)} y_{(3)}=S(x) y_{(1)} \otimes$ $y_{(2)} \otimes y_{(3)}$ and then $x_{(1)} y_{(2)} S^{-1}\left(y_{(1)}\right) \otimes x_{(2)} y_{(3)}=y_{(2)} S^{-1}\left(y_{(1)}\right) x \otimes y_{(3)}$ which shows that $\Delta(x)(\mathbb{1} \otimes y)=x \otimes y$.

Proposition 3.13 Let $P$ be a right group-like projection and $\mathcal{V}_{P} \subseteq \mathcal{N}^{-l p}$ be the left coideal defined in Definition 3.7. Then $\mathcal{V}_{P}$ is a

- $\quad$ right ideal in $\mathcal{N}_{P}^{l}$;

- left ideal in $\mathcal{N}_{P}^{r}$;

- bilateral ideal in $\mathcal{N}_{P}$.

Moreover $\mathcal{V}_{P}$ is idempotent as a (left, right or bilateral) ideal, i.e. $\mathcal{V}_{P}^{2}=\mathcal{V}_{P}$

In particular $\mathcal{V}_{P}=\mathcal{N}_{P}^{- \text {lr }}$ if and only if $\mathbb{1} \in \mathcal{V}_{P}$. In that case we also have

$$
\mathcal{N}_{P}^{l}=\mathcal{N}_{P}=\mathcal{N}_{P}^{r}
$$

Proof That $\mathcal{V}_{P} \subseteq \mathcal{N}_{P}^{l}$ is a right ideal can be seen by applying $\mu \otimes$ id to the fourth bullet point in Lemma 3.12 with $y=P$, where $\mu \in \mathcal{A}^{*}$. The claim that $\mathcal{V}_{P} \subseteq \mathcal{N}_{P}^{r}$ is a left ideal can be seen similarly. $\mathcal{V}_{P}$ is then both a left and a right ideal in $\mathcal{N}_{P}=\mathcal{N}_{P}^{l} \cap \mathcal{N}_{P}^{r}$.

As for the claim that $\mathcal{V}_{P}$ is idempotent, this follows from the fact that it contains $P$ hence so does $\mathcal{V}_{P}^{2}$, meaning that $\mathcal{V}_{P} \subseteq \mathcal{V}_{P}^{2}$.

Finally, if $\mathbb{1} \in \mathcal{V}_{P}$ then $\mathcal{V}_{P}$ is a (left, right, or bilateral) ideal containing the unit in $\mathcal{N}_{P}^{-l r}$ and hence coincides with all of them.

Proposition 3.14 Let $P \in \mathcal{A}$ be a right group-like projection. Then, $\mathcal{V}_{P} \subseteq \mathcal{N}_{P}^{- \text {lr }}$ defined by Eq. 3.9 is faithful as a

- $\quad$ right ideal over $\mathcal{N}_{P}^{l}$;

- left ideal over $\mathcal{N}_{P}^{r}$;

- left or right ideal over $\mathcal{N}_{P}$.

Before moving on to the proof we record a consequence.

Corollary 3.15 For any right group-like projection $P \in \mathcal{A}$ the associated left coideal subalgebras $\mathcal{N}_{P}^{-l r}$ are finite-dimensional.

Proof Indeed, by Proposition 3.14 they act faithfully on a finite-dimensional vector space $\mathcal{V}_{P}$, and hence admit embeddings into the finite-dimensional algebra $\operatorname{End}\left(\mathcal{V}_{P}\right)$.

Proof of Proposition 3.14 To fix ideas and notation we will show that $\mathcal{V}_{P} \subseteq \mathcal{N}_{P}^{r}$ is faithful as a left ideal.

According to Proposition $3.13 \mathcal{V}_{P} \subseteq \mathcal{N}_{P}^{r}$ is an inclusion of left coideal subalgebras of $\mathcal{A}$, and hence the multiplication map

$$
\mathcal{N}_{P}^{r} \otimes \mathcal{V}_{P} \rightarrow \mathcal{V}_{P}
$$

is a morphism of left $\mathcal{A}$-comodules. It induces a morphism

$$
\mathcal{N}_{P}^{r} \rightarrow \mathcal{V}_{P} \otimes \mathcal{V}_{P}^{*} \cong \operatorname{End}\left(\mathcal{V}_{P}\right)
$$


in the same category of $\mathcal{A}$-comodules, and hence its kernel must again be a left $\mathcal{A}$-coideal. In other words, the (two-sided) ideal

$$
\mathcal{V}_{P}^{\perp}:=\left\{x \in \mathcal{N}_{P}^{r} \mid x \mathcal{V}_{P}=0\right\}
$$

is also a left $\mathcal{A}$-coideal. The identity $x_{(1)} \varepsilon\left(x_{(2)}\right)=x$ for $x \in \mathcal{V}_{P}^{\perp}$ then shows that if $\mathcal{V}_{P}^{\perp}$ is non-zero, then it contains elements $y$ such that $\varepsilon(y) \neq 0$.

This leads to a contradiction: on the one hand we have $P \in \mathcal{V}_{P}$ and hence $y P=0$, while on the other hand

$$
y P=\varepsilon(y) P \neq 0
$$

by assumption. It follows that $\mathcal{V} \frac{\perp}{P}=\{0\}$, which is a rephrasing of the conclusion we were after.

Knowing that $\mathcal{N}_{P}$ is finite dimensional we can characterize the cases when $\mathcal{V}_{P}=\mathcal{N}_{P}$. For this purpose following property will be needed.

Definition 3.16 A finite-dimensional algebra is quasi-Frobenius (or QF) if it is injective as a module over itself.

\section{Using [8, Lemma 2.1 and Theorem 2.2] we get}

Theorem 3.17 Let $P \in \mathcal{A}$ be a right group-like projection, $\mathcal{N}_{P}$ the assigned left coideal subalgebra and $\mathcal{V}_{P} \subset \mathcal{N}_{P}$ the left coideal as defined in Definition 3.7. Then $\mathcal{V}_{P}=\mathcal{N}_{P}$ if and only if $\mathcal{N}_{P}$ is quasi-Frobenius.

We do not know an example where $\mathcal{V}_{P} \neq \mathcal{N}_{P}$, but note that the finite-dimensionality of $\mathcal{N}_{P}$ together with the faithfulness result in Proposition 3.14 are not themselves sufficient to conclude this.

Example 3.18 We will describe a finite-dimensional algebra $A$ with a proper idempotent ideal $I$ (i.e. $I^{2}=I$ ) that is both left and right faithful.

$A$ will be spanned by a projection $e$, its complementary projection $1-e$ and the basis $\{x, y, z\}$ for its Jacobson radical, subject to the following conditions:

- $x=\operatorname{ex}(1-e)$;

- $y=(1-e) y e$

- $y x=0$ and $x y=z$;

- $x z=z x=0=y z=z y$;

everything else follows (e.g. $x^{2}=0$ because $\left.(1-e) e=0\right)$.

We set

$$
I=\operatorname{span}\{e, x, y, z\}=A e+e A=A e A,
$$

clearly an idempotent ideal. Checking faithfulness is a simple matter (left to the reader) of writing all five basis elements of $A$ as matrices for the multiplication action and observing that said matrices are linearly independent. In fact it suffices to check this for left multiplication, since the map interchanging $x$ and $y$ and fixing $e, z$ is an involutive anti-automorphism of $A$ fixing $I$.

Definition 3.19 An algebra $R$ is weakly finite if $x y=1 \Rightarrow y x=1$ for all $x, y \in M_{n}(R)$ and all positive integers $n$. 
[17, Theorem 6.1] shows that finite-dimensional left coideal subalgebras in weakly finite Hopf algebras are automatically Frobenius (and hence also QF). Therefor using Theorem 3.17 we get the next result.

Corollary 3.20 Let $\mathcal{A}$ be a weakly finite Hopf algebra (e.g. $\mathcal{A}$ is left or right noetherian) and $P \in \mathcal{A}$ a right group-like projection. Then $\mathcal{N}_{P}=\mathcal{V}_{P}$.

The results of [8] allows us also to deduce certain semisimplicity result about $\mathcal{N}_{P}$. Before formulating it let us prove the following proposition.

Proposition 3.21 Let $P \in \mathcal{A}$ be right group-like projection. Then the following are equivalent:

- $\quad P$ is a two sided group-like projection;

- we have $S^{2}\left(\mathcal{N}_{P}\right)=\mathcal{N}_{P}$;

- we have $\Delta(P) \in S\left(\mathcal{N}_{P}\right) \otimes \mathcal{N}_{P}$

Moreover, if $P$ is two-sided then $\mathcal{V}_{P}=\mathcal{N}_{P}^{-l r}$.

Proof Suppose that $P$ is a right group-like projection. Note that $S^{2}(P)$ is a right group-like projection and $S^{2}\left(\mathcal{N}_{P}\right)=\mathcal{N}_{S^{2}(P)}$. In particular $S^{2}\left(\mathcal{N}_{P}\right)=\mathcal{N}_{P}$ if and only if $P=S^{2}(P)$ (c.f. Proposition 3.8) and this by Theorem 3.5 holds if and only if $P$ is also a left group-like projection.

Now, if $P$ is a two sided group-like projection then $S(P)=P$ and it is easy to check that $\Delta(P) \subset S\left(\mathcal{N}_{P}\right) \otimes \mathcal{N}_{P}$. Conversely if $P$ is a right group-like projection and $\Delta(P) \in$ $S\left(\mathcal{N}_{P}\right) \otimes \mathcal{N}_{P}$ then $\Delta(P)(S(P) \otimes \mathbb{1})=S(P) \otimes P=(S(P) \otimes \mathbb{1}) \Delta(P)$. Applying id $\otimes \varepsilon$ we get $P S(P)=S(P) P=S(P)$ and we conclude that $P$ is a two sided group-like projection using Theorem 3.5.

In order to prove the last claim of the proposition it is enough to show that $\mathbb{1} \in \mathcal{V}_{P}$. Using Sweedler's notation $\Delta(P)=P_{(1)} \otimes P_{(2)}$ and we have $P_{(1)} \in S\left(\mathcal{V}_{P}\right)$ and $P_{(2)} \in \mathcal{V}_{P}$. In particular $\mathbb{1}=S\left(P_{(1)}\right) P_{(2)} \in \mathcal{V}_{P}$ and we are done.

Using [8, Propositions 5.1 and 5.2] and Proposition 3.21we get the next result.

Theorem 3.22 If $P$ be a right group-like projection in $\mathcal{A}$, then $\mathcal{N}_{P}$ is semisimple if and only if $\mathcal{N}_{P}$ is a direct summand of $\mathcal{A}$ viewed as a $\mathcal{N}_{P}$ two-sided module.

If $P$ is a two sided group-like projection in $\mathcal{A}$, then $\mathcal{N}_{P}$ is semisimple.

Proposition 3.14 allows us to give the following partial characterization of coideal subalgebras of the form $\mathcal{N}_{P}$.

Proposition 3.23 Suppose the coideal subalgebra $\mathcal{N} \subset \mathcal{A}$ is semisimple. Then, $\mathcal{N}=\mathcal{N}_{P}$ for some right group-like projection $P$ and $\mathcal{N}_{P}=\mathcal{V}_{P}$.

Proof The semisimplicity of $\mathcal{N}$ means that $\operatorname{ker}\left(\left.\varepsilon\right|_{\mathcal{N}}\right)$ is of the form $\mathcal{N}(1-P)$ for a projection $P$ which is central in $\mathcal{N}$, i.e. $\mathcal{N}$ automatically has an integral $P$. We then have

$$
\mathcal{V}_{P} \subseteq \mathcal{N} \subseteq \mathcal{N}_{P}
$$


and it suffices to show that $\mathcal{V}_{P}=\mathcal{N}$ (for then $\mathbb{1} \in \mathcal{V}_{P}$ and hence the inclusions in Eq. 3.11 are all equalities).

Finally, to prove $\mathcal{V}_{P}=\mathcal{N}$ recall that the former is a faithful ideal in the latter by Proposition 3.14; since ideals in semisimple algebras are generated by central projections they cannot be (either left or right) faithful if proper. We thus have $\mathcal{V}_{P}=\mathcal{N}_{P}=\mathcal{N}$, as claimed.

Finally, the results gathered thus far suffice to identify two-sided group-like projections as those giving rise to a certain class of coideal subalgebras. For locally compact quantum group the analog of the next result was obtained in [3]. It should also be compared with [8, Proposition 5.2].

Theorem 3.24 Let $\mathcal{A}$ be an arbitrary Hopf algebra. The correspondence $P \mapsto \mathcal{N}_{P}$ induces a bijection between the set of two-sided group-like projections and the set of semisimple coideal subalgebras of $\mathcal{A}$ preserved by the squared antipode.

Proof The map $P \mapsto \mathcal{N}_{P}$ does indeed land in the set of semisimple coideal subalgebras fixed by $S^{2}$ by Theorem 3.22. It is one-to-one by Proposition 3.21 and onto by Proposition 3.23.

\subsection{Miscellaneous Results}

In this subsection we shall prove some loosely related results concerning group-like projections, coideals, faithful flatness, Hopf subalgebras etc.

Proposition 3.25 Let $P$ be a right group-like projection. Then $\mathcal{N}_{P}$ forms a Hopf subalgebra if and only if $\Delta(P) \in \mathcal{N}_{P} \otimes \mathcal{N}_{P}$. In particular $P$ is two sided group-like projection in this case.

Proof The leftward implication is obvious.

Suppose conversely that $\Delta(P) \in \mathcal{N}_{P} \otimes \mathcal{N}_{P}$. Then clearly

$$
\Delta(P)(P \otimes \mathbb{1})=P \otimes P=(P \otimes \mathbb{1}) \Delta(P)
$$

i.e. $P$ is two sided. Using Proposition 3.21 we get $\mathcal{N}_{P}=\left\{(\mu \otimes\right.$ id $\left.)(\Delta(P)): \mu \in \mathcal{A}^{*}\right\}$ and thus $S\left(\mathcal{N}_{P}\right)=\left\{(\operatorname{id} \otimes \mu)(\Delta(P)): \mu \in \mathcal{A}^{*}\right\} \subset \mathcal{N}_{P}$; similarly $S^{-1}\left(\mathcal{N}_{P}\right)=\{(\operatorname{id} \otimes \mu)(\Delta(P))$ : $\left.\mu \in \mathcal{A}^{*}\right\} \subset \mathcal{N}_{P}$ and both containments together yield the equality $\mathcal{N}_{P}=S\left(\mathcal{N}_{P}\right)$. We conclude by noting that a left coideal subalgebra preserved by $S$ must be a Hopf algebra.

Proposition 3.26 Let $P \in \mathcal{A}$ be central group-like projection. Then $\mathcal{N}_{P}$ is a normal left coideal subalgebra and $P$ is a two sided counital integral in $\mathcal{N}_{P}$. Conversely, let $\mathcal{N}$ be a normal left coideal with a counital two sided integral $P \in \mathcal{N}$. Then $P$ is central in $\mathcal{A}$ and $\mathcal{N}=\mathcal{N}_{P}$

Proof Suppose that $P$ is a central right group-like projection and $x \in \mathcal{N}_{P}$. Then for every $a \in \mathcal{A}$ we have

$$
\begin{aligned}
\Delta\left(a_{(1)} x S\left(a_{(2)}\right)\right)(\mathbb{1} \otimes P) & =a_{(1)} x_{(1)} S\left(a_{(4)}\right) \otimes a_{(2)} x_{(2)} S\left(a_{(3)}\right) P \\
& =a_{(1)} x_{(1)} S\left(a_{(4)}\right) \otimes a_{(2)} x_{(2)} P S\left(a_{(3)}\right) \\
& =a_{(1)} x S\left(a_{(4)}\right) \otimes a_{(2)} P S\left(a_{(3)}\right)=a_{(1)} x S\left(a_{(2)}\right) \otimes P
\end{aligned}
$$


and we see that $\mathcal{N}_{P}$ is preserved by the adjoint action. Clearly $P$ is an integral for $\mathcal{N}_{P}$.

Conversely, suppose that $\mathcal{N}$ is a normal coideal and $P \in \mathcal{N}$ is an integral. Then for every $a \in \mathcal{A}$ we have

$$
a_{(1)} P S\left(a_{(2)}\right) P=\varepsilon\left(a_{(1)} P S\left(a_{(2)}\right)\right) P=\varepsilon(a) P
$$

and similarly we get

$$
P a_{(1)} P S\left(a_{(2)}\right)=\varepsilon(a) P .
$$

Viewing $P$ as a left multiplication operator on $\mathcal{A}$ we have

$$
\begin{aligned}
\mathrm{W}_{l r}(\mathbb{1} \otimes P) \mathrm{W}_{l r}^{-1}(\mathbb{1} \otimes P)(a \otimes b) & =\mathrm{W}_{l r} a_{(1)} \otimes P S\left(a_{2}\right) P b \\
& =a_{(1)} \otimes a_{(2)} P S\left(a_{3}\right) P b \\
& =a \otimes P b
\end{aligned}
$$

where in the third equality we used (3.12). This shows that $\mathrm{W}_{l r}(\mathbb{1} \otimes P) \mathrm{W}_{l r}^{-1}(\mathbb{1} \otimes P)=\mathbb{1} \otimes P$. Similarly we get $(\mathbb{1} \otimes P) \mathrm{W}_{l r}(\mathbb{1} \otimes P) \mathrm{W}_{l r}^{-1}=(\mathbb{1} \otimes P)$. Using $(\mathbb{1} \otimes P) \mathrm{W}_{l r}^{-1}(\mathbb{1} \otimes P)=$ $\mathrm{W}_{l r}^{-1}(\mathbb{1} \otimes P)$ we see that

$$
a_{1} \otimes P S\left(a_{(2)}\right) P b=a_{1} \otimes S\left(a_{(2)}\right) P b
$$

for every $a, b \in \mathcal{A}$. Applying $\varepsilon \otimes$ id and putting $b=\mathbb{1}$ we get $P S(a) P=S(a) P$ for every $a \in \mathcal{A}$. Similar reasoning starting with $(\mathbb{1} \otimes P) \mathrm{W}_{l r}(\mathbb{1} \otimes P) \mathrm{W}_{l r}^{-1}=(\mathbb{1} \otimes P)$ yields $P a P=P a$. Thus $P a=P a P=P S\left(S^{-1}(a)\right) P=S\left(S^{-1}(a)\right) P=a P$ for all $a \in \mathcal{A}$ and we see that $P$ is central. Using Theorem 3.5 we conclude that $P$ is a two-sided group-like projection and using Proposition 3.21 for the equality below we have

$$
\mathcal{V}_{P} \subseteq \mathcal{N} \subseteq \mathcal{N}_{P}=\mathcal{V}_{P}
$$

and $\mathcal{N}=\mathcal{N}_{P}$

Central group-like projections for locally compact quantum groups were considered in [4] in relations with open quantum subgroups. In our case we have:

Corollary 3.27 Let $P \in \mathcal{A}$ be a right group-like projection such that $\mathcal{N}_{P}$ is normal. Then there is a Hopf algebra $\mathcal{B}$ and a Hopf surjection $\pi: \mathcal{A} \rightarrow \mathcal{B}$ such that $\mathcal{N}_{P}=\{x \in \mathcal{A}$ : $(\mathrm{id} \otimes \pi) \Delta(a)=a \otimes \mathbb{1}\}$.

Proof Centrality of $P$ and Theorem 3.5 yields $S(P)=P$. We conclude by defining $\mathcal{B}=$ $\mathcal{A} P, \Delta_{\mathcal{B}}(b)=\Delta_{\mathcal{A}}(b)(P \otimes P), S_{\mathcal{B}}=\left.S_{\mathcal{A}}\right|_{\mathcal{B}}$ and $\pi(a)=a P$ for all $a \in \mathcal{A}$ and $b \in \mathcal{B}$.

Let us recall that $\mathcal{N}_{P}$ is a left coideal subalgebra containing a projection $P$ satisfying $P x=\varepsilon(x) P=x P$ for all $x \in \mathcal{N}_{P}$. In the next proposition we get a converse to this fact for two sided group-like projections, which is the Hopf algebraic analog of [7, Theorem 4.1.].

Proposition 3.28 Let $\mathcal{N} \subset \mathcal{A}$ be a coideal subalgebra admitting a non-zero projection $Q \in \mathcal{N}$ and a homomorphism $\mu: \mathcal{N} \rightarrow \mathbb{k}$ such that $Q x=x Q=\mu(x) Q$ for every $x \in \mathcal{N}$. If $S^{2}(Q)=Q$ then there exists a two sided group-like projection such that $\mathcal{N}=\mathcal{N}_{P}$ and we have $\mathcal{N}=\left\{(v \otimes \mathrm{id})(\Delta(Q)): v \in \mathcal{A}^{*}\right\}$. Moreover $Q$ is a left shift of $P$.

Proof We denote $\pi=($ id $\otimes \mu) \circ \Delta: \mathcal{N} \rightarrow \mathcal{A}$. Note that $\Delta(a)(\mathbb{1} \otimes Q)=\pi(a) \otimes Q$. It is easy to check that $\pi$ is an injective algebra homomorphism such that $\Delta \circ \pi=(\operatorname{id} \otimes \pi) \circ \Delta$. In particular $\pi(\mathcal{N})$ is a left coideal subalgebra and it contains a two sided group-like projection 
$\pi(Q)$ (see Lemma 2.2), thus $\mathcal{V}_{\pi(Q)} \subset \pi(\mathcal{N})$. Moreover for every $b \in \pi(\mathcal{N})$ there exists $c \in \mathcal{A}$ such that $\Delta(b)(\mathbb{1} \otimes \pi(Q))=c \otimes \pi(Q)$ and since $\varepsilon(\pi(Q))=\mathbb{1}$ we get $b=c$. This shows that $\pi(\mathcal{N}) \subset \mathcal{N}_{\pi(Q)}$. Since $\pi(Q)$ is two sided we have $\mathcal{N}_{\pi(Q)}=\mathcal{V}_{\pi(Q)}=\pi(\mathcal{N})$ and thus $\mathcal{N}=\left\{(\mu \otimes \mathrm{id})(\Delta(Q)): \mu \in \mathcal{A}^{*}\right\}$. Using $\Delta(Q)(\mathbb{1} \otimes Q)=\pi(Q) \otimes Q$ and Proposition 3.6 we can find a two sided group-like projection $P$ such that $\Delta(Q)(Q \otimes \mathbb{1})=Q \otimes P$. In particular $P \in \mathcal{N}$ and thus $\mathcal{N}_{P} \subset \mathcal{N}$. On the other hand since $Q \otimes Q=S^{-1}\left(P_{(1)}\right) Q \otimes P_{(2)}$ we also get $Q \in \mathcal{N}_{P}$ which implies that $\mathcal{N} \subset \mathcal{N}_{P}$ and we are done.

Remark 3.29 Using [17] we conclude that if $\mathcal{A}$ is weakly finite Hopf algebra $\mathcal{A}$ then $\mathcal{A}$ is $\mathcal{N}_{P}$-faithfully flat for every right group-like projection $P \in \mathcal{A}$ and in particular

$$
\mathcal{N}_{P}=\{x \in \mathcal{A}:(\mathrm{id} \otimes \pi)(\Delta(x))=x \otimes \pi(\mathbb{1})\}
$$

where $\pi: \mathcal{A} \rightarrow \mathcal{C}_{\mathcal{N}_{P}}$ is the quotient map (see the first paragraph of Section 2 for more explanations).

$\mathcal{N}_{P}$-faithful flatness is also guarantied if $\mathcal{N}_{P}$ is semisimple, which is the case if e.g. $P$ is a two sided group-like projection (see Theorem 3.2). But in general we do not know if $\mathcal{A}$ is $\mathcal{N}_{P}$-faithfully flat, and we do not know if Eq. 3.14 holds.

In order to relate in Proposition 3.30 the right hand side of Eq. 3.14 to $\mathcal{N}_{P}$ in possibly non $\mathcal{N}_{P}$-faithfully flat case, we shall still denote $\pi: \mathcal{A} \rightarrow \mathcal{C}_{\mathcal{N}_{P}}$ and we denote

$$
\mathcal{N}_{\pi}=\{x \in \mathcal{A}:(\mathrm{id} \otimes \pi)(\Delta(x))=x \otimes \pi(\mathbb{1})\} .
$$

Consider also the left $\mathcal{A}$ module $\mathcal{A} P$. Equipping $\mathcal{A} P$ with the coalgebra structure $x P \mapsto$ $\Delta(x P)(P \otimes P)$ we see that $\mathcal{A} P$ has a structure of a left module quotient coalgebra.

Proposition 3.30 Let $P \in \mathcal{A}$ be a right group-like projection, $\mathcal{N}_{P}$ the left coideal subalgebra assigned to $P$ and $\mathcal{C}_{\mathcal{N}_{P}}$, AP the module quotient coalgebras described in Remark 3.29. Let $\pi: \mathcal{A} \rightarrow \mathcal{C}_{\mathcal{N}_{P}}$ be the canonical quotient maps. Then $\left.\pi\right|_{\mathcal{A} P}$ identifies $\mathcal{A} P$ with $\mathcal{C}_{\mathcal{N}_{P}}$ and we have $\mathcal{N}_{\pi}=\mathcal{N}_{P}^{r}$. In particular if $\mathcal{N}_{P}=\mathcal{V}_{P}$ then $\mathcal{N}_{P}=\mathcal{N}_{\pi}$.

Proof Since $\mathbb{1}-P \in \mathcal{N}_{P}^{-}$we have $\pi(\mathbb{1})=\pi(P)$. In particular, if $x \in \mathcal{A}$ then $\pi(x)=$ $x \pi(\mathbb{1})=x \pi(P)=\pi(x P)$ and thus $\left.\pi\right|_{\mathcal{A} P}$ is onto. If in turn $\pi(x P)=0$ then $0=x \pi(P)=$ $x \pi(\mathbb{1})=\pi(x)$ and hence $x \in \mathcal{A N}_{P}^{-}$. In particular $x P \in \mathcal{A N}_{P}^{-} P=\{0\}$ and we see that $\left.\pi\right|_{\mathcal{A} P}$ is isomorphism of left $\mathcal{A}$ modules. Finally, the equality $\Delta(x P)(P \otimes P)=\Delta(x)(P \otimes$ $P$ ) shows that $\left.\pi\right|_{\mathcal{A} P}$ identifies $\mathcal{A} P$ and $\mathcal{C}_{\mathcal{N}_{P}}$ as module quotient coalgebras. Under this identification the quotient map becomes $\pi(x)=x P$ which immediately implies that

$$
\mathcal{N}_{\pi}=\{x \in \mathcal{A}: \Delta(x)(\mathbb{1} \otimes P)=x \otimes P\}=\mathcal{N}_{P}^{r} .
$$

Using Proposition 3.13 we see that $\mathcal{N}_{P}=\mathcal{N}_{\pi}$ if $\mathcal{N}_{P}=\mathcal{V}_{P}$.

Remark 3.31 Let $P \in \mathcal{A}$ be a projection in $\mathcal{A}$ satisfying $\Delta(P)(P \otimes P)=P \otimes P$ and consider the left $\mathcal{A}$ module $\mathcal{C}_{P}=\mathcal{A} P$. Let us define $\Delta_{\mathcal{C}_{P}}: \mathcal{C}_{P} \rightarrow \mathcal{C}_{P} \otimes \mathcal{C}_{P}$ by $\Delta_{\mathcal{C}_{P}}(x)=$ $\Delta(x)(P \otimes P)$. It is easy to see that that $\Delta_{\mathcal{C}_{P}}$ is coassociative. Moreover if $\varepsilon(P)=1$ then $\left(\mathcal{C}_{P}, \Delta_{\mathcal{C}_{P}},\left.\varepsilon\right|_{\mathcal{C}_{P}}\right)$ together with the map $\pi: \mathcal{A} \rightarrow \mathcal{C}$ given by $\pi(x)=x P$ is a left module quotient coalgebra. Clearly $\mathcal{N}_{\pi}=\{x \in \mathcal{A}: \Delta(x)(\mathbb{1} \otimes P)=x \otimes P\}$ thus $P \in \mathcal{N}_{\pi}$ if and only $\Delta(P)(\mathbb{1} \otimes P)=P \otimes P$. Moreover if $P \in \mathcal{N}_{\pi}$ then $x P=\varepsilon(x) P$ for all $x \in \mathcal{N}_{\pi}$.

In what follows we shall show that $\mathcal{C}_{P}$ can be identified with $\mathcal{C}_{\mathcal{N}_{\pi}}$ if $P \in \mathcal{A}$ is a projection such that $\Delta(P)(\mathbb{1} \otimes P)=P \otimes P$. Clearly such a projection satisfies $\Delta(P)(P \otimes P)=P \otimes P$ and $\varepsilon(P)=1$ and it makes sense to consider a left module quotient coalgebra $\mathcal{C}_{P}$ in the first place. In order to identify $\mathcal{C}_{P}$ and $\mathcal{C}_{\mathcal{N}_{\pi}}$ we must show that $\mathcal{A N}_{\pi}^{-}=\mathcal{A}(\mathbb{1}-P)$. The 
containment $\mathcal{A}(\mathbb{1}-P) \subset \mathcal{A} \mathcal{N}_{\pi}^{-}$is clear. For the converse containment we have $\mathcal{A} \mathcal{N}_{\pi}^{-}=$ $\mathcal{A N}_{\pi}^{-}(\mathbb{1}-P) \subset \mathcal{A}(\mathbb{1}-P)$.

Definition 3.32 A projection $P \in \mathcal{A}$ satisfying and $\varepsilon(P)=\mathbb{1}$ is called

- $\quad$ a right sided weak group-like projection if $\Delta(P)(P \otimes P)=P \otimes P$;

- a left sided weak group-like projection if $(P \otimes P) \Delta(P)=P \otimes P$;

- a weak group-like projection if $\Delta(P)(P \otimes P)=(P \otimes P) \Delta(P)=P \otimes P$.

Clearly a right (left) group-like projection is also a weak group-like projection. In Remark 4.11 we shall see that the number of weak group-like projections in Sweedler's Hopf algebra is substantially larger the the number of right (left) group-like projections.

Motivated by [5, Theorem 4.5] we prove the next result.

Proposition 3.33 Let $P \in \mathcal{A}$ be a right sided weak group-like projection $\Delta(P)(P \otimes P)=$ $P \otimes P$ and $S(P)=P$. Then $P$ is a group-like projection.

Proof For the needs of the Sweedler's notation we shall denote $P=\tilde{P}$ when necessary. Using $P_{(1)} \tilde{P} \otimes P_{(2)} \tilde{P}=P \otimes P$ we get

$$
P_{(1)} \tilde{P}_{(1)} \otimes S\left(\tilde{P}_{(2)}\right) S\left(P_{(2)}\right) P_{(3)} \tilde{P}=P_{(1)} \otimes S\left(P_{(2)}\right) P .
$$

Noting that the left hand side is equal $P P_{(1)} \otimes S\left(P_{(2)}\right) P$ we conclude that (note that tildas are dropped)

$$
S\left(P_{(1)}\right) S(P) \otimes S\left(P_{(2)}\right) P=S\left(P_{(1)}\right) \otimes S\left(P_{(2)}\right) P
$$

Now using $S(P)=P$ we get $\Delta^{\mathrm{op}}(P)(\mathbb{1} \otimes P)=P \otimes P$.

Since $\Delta^{\mathrm{op}}(P)(P \otimes P)=P \otimes P$ we can apply the same reasoning to $\Delta^{\text {op }}$ to get $\Delta(P)(\mathbb{1} \otimes$ $P)=P \otimes P$. Using Theorem 3.5 we see that $P$ is a two sided group-like projection.

\subsection{Semisimple Hopf Algebras}

Throughout the present section $\mathcal{A}$ denotes a semisimple Hopf algebra over $\mathbb{k}$.

Lemma 3.34 An arbitrary coideal subalgebra $\mathcal{N} \subseteq \mathcal{A}$ is of the form $\mathcal{N}_{P}$ for some right group-like projection $P$.

Proof Since $\mathcal{A}$ is semisimple, so is $\mathcal{N}$ (e.g. [1, Lemma 4.0.2] or [17, Theorem 5.2]). Now simply apply Proposition 3.23.

All in all, the conclusion is that right group-like projections are an alternate manifestation of coideal subalgebras, at least in semisimple Hopf algebras, as the following one-sided analogue of Theorem 3.24 confirms.

Theorem 3.35 Let $\mathcal{A}$ be a semisimple Hopf algebra. The maps

$$
\mathcal{N} \mapsto P_{\mathcal{N}}:=\text { the central projection satisfying } \operatorname{ker}\left(\left.\varepsilon\right|_{\mathcal{N}}\right)=(\mathbb{1}-P) \mathcal{N}
$$

and $P \mapsto \mathcal{N}_{P}$ are mutually inverse bijections between the set of coideal subalgebras of $\mathcal{A}$ and its set of right group-like projections. 
Proof Lemma 3.34 verifies that

$$
\mathcal{N} \mapsto P_{\mathcal{N}} \mapsto \mathcal{N}_{P_{\mathcal{N}}}
$$

is the identity. On the other hand, consider a right group-like projection $P$. Its associated coideal subalgebra $\mathcal{N}=\mathcal{N}_{P}$ has an attached projection $Q=P_{\mathcal{N}_{P}}$. Lemma 3.34 once more shows that $\mathcal{N}_{Q}=\mathcal{N}=\mathcal{N}_{P}$, meaning that $Q=P$ by Proposition 3.21.

Remark 3.36 Using [11, Theorem 3] and Theorem 3.5 we see that right group-like projections are two sided group-like projections if $\mathcal{A}$ in a simple Hopf algebra over a field of characteristic $p>\operatorname{dim}(H)^{2}$.

In fact, more generally, if the Hopf algebra is simultaneously semisimple and cosemisimple then its antipode is an involution [2]; it then again follows from Theorem 3.5 that right group-like projections are two-sided.

Remark 3.37 Let $\mathcal{A}$ be a finite dimensional Hopf algebra and $\mathcal{N}$ a left coideal subalgebra. Let $\pi: \mathcal{A} \rightarrow \mathcal{C}_{\mathcal{N}}$ be the assigned left module quotient coalgebra as described in Remark 3.29. Then $\pi^{*}\left(\mathcal{C}^{*}\right) \subset \mathcal{A}^{*}$ forms a left coideal subalgebra. Using [17, Corollary 6.5] we see that $\mathcal{N} \mapsto \pi^{*}\left(\mathcal{C}_{\mathcal{N}}^{*}\right)$ establishes a bijective correspondence between left coideal subalgebras of $\mathcal{A}$ and $\mathcal{A}^{*}$. Let us note that $\pi^{*}\left(\mathcal{C}_{\mathcal{N}}^{*}\right)$ is of the form $\mathcal{N}_{\tilde{P}}$ for some right group like projection $\tilde{P} \in \mathcal{A}^{*}$ if and only if $\pi^{*}\left(\mathcal{C}_{\mathcal{N}}^{*}\right)$ admits a two sided counital integral in the sense of Definition 3.10. Indeed, in this case

$$
\mathcal{V}_{\tilde{P}} \subseteq \pi^{*}\left(\mathcal{C}_{\mathcal{N}}^{*}\right) \subseteq \mathcal{N}_{\tilde{P}}=\mathcal{V}_{\tilde{P}}
$$

where the in last equality we use Corollary 3.20. Clearly $\pi^{*}\left(\mathcal{C}_{\mathcal{N}}^{*}\right)$ admits an integral if and only if there exists a functional $\psi \in \mathcal{C}_{\mathcal{N}}^{*}$ such that $(\psi \otimes \mathrm{id})\left(\Delta_{\mathcal{C}_{\mathcal{N}}}(x)\right)=(\mathrm{id} \otimes$ $\psi)\left(\Delta_{\mathcal{C}_{\mathcal{N}}}(x)\right)=\psi(x) \pi(\mathbb{1})$ for all $x \in \mathcal{C}_{\mathcal{N}}$.

Suppose that $\mathcal{A}$ is a semisimple and cosemisimple Hopf algebra. Then $\mathcal{A}^{*}$ is also semisimple and cosemisimple. Since in this case the antipode is involutive, then using Theorem 3.35 and the above considerations we get a bijective correspondence between group-like projections in $\mathcal{A}$ and $\mathcal{A}^{*}$.

\section{Taft Algebras}

The purpose of the present section is to classify the left coideal subalgebras of a certain family of Hopf algebras, identifying among them those that correspond to right group-like projections via the construction $P \mapsto \mathcal{N}_{P}$ from Eq. 3.8.

Following [19], we have

Definition 4.1 Let $n$ be a positive integer and $\omega \in \mathbb{k}$ a primitive $n^{\text {th }}$ root of unity. The Taft Hopf algebra $H_{n^{2}}$ is the algebra $\mathbb{k}[x] /\left(x^{n}\right) \rtimes \mathbb{k}[G]$, where $G=\mathbb{Z} / n \mathbb{Z}$ is generated by $g$ and its action on the truncated polynomial ring $\mathbb{k}[x] /\left(x^{n}\right)$ is by

$$
g x g^{-1}=\omega x \text {. }
$$

The coalgebra structure is given by

$$
\Delta(g)=g \otimes g, \quad \Delta(x)=x \otimes 1+g \otimes x .
$$

These Hopf algebras appear frequently in the literature, being $\omega$-deformed analogues of the enveloping algebra of the Borel subalgebra $\mathfrak{b} \subset \mathfrak{s l}_{2}$. See e.g. $[12,14,15]$. They were 
originally introduced in [19] as part of a larger family meant to show that finite-dimensional Hopf algebras can have antipodes of arbitrary even order (for $H_{n^{2}}$ the antipode order is $2 n$ ).

The notation $H_{n^{2}}$ is meant to remind the reader that $H$ so defined is $n^{2}$-dimensional, for instance with a basis consisting of

$$
x^{i} g^{j}, 0 \leq i, j \leq n-1 .
$$

Throughout this section we focus on the case when $n$ is prime, this assumption being henceforth implicitly in place unless specified otherwise. Moreover, since we are assuming that $k$ contains primitive $n^{\text {th }}$ roots of unity, the characteristic of $\mathbb{k}$ is coprime to $n$ and hence $\mathbb{k} G$ is semisimple.

Let $\mathcal{N} \subset H=H_{n_{2}}$ be a proper, non-trivial left coideal subalgebra. $\mathcal{N}$ further maps through

$$
\mathcal{N} \rightarrow H \rightarrow \mathbb{k}_{k} G
$$

to a coideal subalgebra of $\mathbb{k}_{k} G$. The latter are simply the subalgebras of the form $\mathbb{k}_{k} G^{\prime}$ for subgroups $G^{\prime} \leq G$, and hence are automatically semisimple. On the other hand, the kernel of Eq. 4.1 consists of nilpotent elements. In conclusion, the kernel of Eq. 4.1 is precisely the Jacobson radical $J(\mathcal{N})$ while its image is the semisimple quotient $\mathcal{N}_{s s}$ of $\mathcal{N}$.

We have two possibilities:

- $\quad \mathcal{N}$ is semisimple, meaning that Eq. 4.1 is one-to-one;

- it is not, i.e. $J(N)$ is non-zero.

$H$ is naturally $\mathbb{Z}$-graded by

$$
\operatorname{deg} g=0, \operatorname{deg} x=1 .
$$

We denote the degree- $d$ component of $H$ by $H_{d}$. Equipped with the grading just defined $H$ is a graded Hopf algebra in the sense of [13, Definition 10.5.11]: it is a graded algebra as well as a graded coalgebra, i.e.

$$
\Delta\left(H_{d}\right) \subset \bigoplus_{i} H_{i} \otimes H_{d-i}
$$

We will often refer to the degree of a possibly-non-homogeneous element $y$ of $H$, meaning the largest degree $i$ of an element $x^{i} g^{j}$ appearing in an expansion of $y$ in the basis $\left\{x^{i} g^{j}\right\}$. We write $\operatorname{deg}(y)$ for the degree in this sense, as well as $\operatorname{deg}^{\prime}(y)$ for the smallest degree of a summand of $y$. The free term of $y$ is the sum of all of its degree-zero summands; equivalently, it is the image of $y$ through the surjection $H \rightarrow \mathbb{k} G$. It is non-zero precisely when $\operatorname{deg}^{\prime}(y)=0$.

We now start to analyze the structure of the coideal subalgebras of $H$.

Lemma 4.2 Let $n \geq 2$ be an arbitrary positive integer and $\mathcal{N} \subset H=H_{n^{2}}$ a non-trivial proper left coideal subalgebra.

If $\mathcal{N}$ contains a non-zero element $y$ with no free term then it contains, for each $1 \leq k \leq$ $n-1$, an element of the form $x^{k} g^{i}$ for some $0 \leq i \leq n-1$ (possibly dependent on $k$ ).

Proof Let

where $y_{i} \in H_{i}$ and

$$
y=y_{d^{\prime}}+\cdots+y_{d}
$$

$$
d=\operatorname{deg}(y), 0<d^{\prime}=\operatorname{deg}^{\prime}(y) .
$$

Because $\mathcal{N}$ is a left coideal subalgebra the elements

$$
\varphi\left(y_{(1)}\right) y_{(2)}, \varphi \in H^{*}
$$


are all contained in $\mathcal{N}$. Choosing $\varphi$ in the summand $H_{1}^{*}$ of $H^{*}$ will produce an element $z \in \mathcal{N}$ with $\operatorname{deg}^{\prime}(z)=\operatorname{deg}^{\prime}(y)-1=d^{\prime}-1$. Applying this repeatedly if necessary we obtain elements $z \in \mathcal{N}$ with $\operatorname{deg}^{\prime}(z)=1$. We thus discard the symbol ' $z$ ' and assume $d^{\prime}=1$ throughout the rest of the proof.

Writing $y_{1}$ as $\sum a_{j} x g^{j}$ for $0 \leq j \leq n-1$ we have

$$
y_{1(1)} \otimes y_{1(2)}=\sum a_{j}\left(x g^{j} \otimes g^{j}+g^{j+1} \otimes x g^{j}\right)
$$

Applying to this element a functional of the form $\varphi \otimes$ id for $\varphi \in H_{0}^{*}$ annihilating all $g^{\ell}$ save for a single $g^{j+1}$ with $a_{j} \neq 0$ we obtain an element $z \in \mathcal{N}$ with $\operatorname{deg}^{\prime}(z)=1$ and $z_{1}$ being a single element $x g^{j}$ of the standard basis of $H$.

We now have

$$
x^{n-1} g^{j(n-1)}=z_{1}^{n-1}=z^{n-1} \in \mathcal{N},
$$

i.e. $\mathcal{N}$ contains monomials of top degree $n-1$. We re-purpose ' $j$ ' and simply write $x^{n-1} g^{j} \in \mathcal{N}$. The $H_{n-2} \otimes H_{1}$-component of $\Delta\left(x^{n-1} g^{j}\right) \in H \otimes \mathcal{N}$ is

$$
\left(1+\cdots+\omega^{n-2}\right) x^{n-2} g^{j+1} \otimes x g^{j}=-\omega^{n-1} x^{n-2} g^{j+1} \otimes x g^{j} \neq 0
$$

so the right hand tensorand $x g^{j}$ belongs to $\mathcal{N}$. Finally, the powers of this element satisfy the desired conclusion.

This will allow us to better understand the coideal subalgebras falling in the second of the two qualitative classes noted in the discussion preceding Lemma 4.2.

Proposition 4.3 The non-semisimple coideal subalgebras $\mathcal{N} \subset H$ are $\mathcal{N}_{d, x}$ for various divisors $d \mid n$, defined by

$$
\mathcal{N}_{d, x}:=\text { algebra generated by } g^{d} \text { and } x .
$$

Proof It is immediate that $\mathcal{N}_{d, x}$ is indeed a coideal subalgebra (it is the algebra generated by the coideals $\operatorname{span}\{1, x\}$ and $\mathbb{k}^{d}$ ). We now have to show that an arbitrary (non-semisimple) $\mathcal{N}$ is of the form $\mathcal{N}_{d, x}$ for some $d \mid n$.

First, we identify $d$ : the intersection $\mathcal{N} \cap \mathbb{k} G$ is of the form $\mathbb{k} G^{\prime}$ for some subgroup $G^{\prime} \leq G$, and we set $d=\left|G / G^{\prime}\right|$. Now let $y \in \mathcal{N}$ be an element of degree $\ell>0$ whose maximal-degree component $y_{\ell}$ contains a scalar multiple of some $x^{\ell} g^{j}$.

Choose some $\varphi \in H^{*}$ that annihilates all $x^{i} g^{j}$ except for $i=\ell$. Applying $\varphi \otimes$ id to $\Delta(y)$ produces a non-zero scalar multiple of $g^{j}$, which must belong to $\mathcal{N}$ because the latter is a left coideal. Our definition of $d$ entails $d \mid j$. In conclusion, for all $y \in \mathcal{N}$ the top-degree component $y_{\ell}$ belongs to $\mathcal{N}_{d, x}$; by induction on degree we obtain $\mathcal{N} \subseteq \mathcal{N}_{d, x}$.

Conversely, $\mathcal{N}$ contains $G^{\prime}=\left\{g^{k d} \mid k \in \mathbb{Z}\right\}$ as well as $x g^{j}$ for some $j$ by Lemma 4.2. We already know from the preceding discussion that $d \mid j$, so some element $\left(x g^{j}\right)\left(g^{d}\right)^{k} \in \mathcal{N}$ equals $x$. This proves the opposite inclusion $\mathcal{N} \supseteq \mathcal{N}_{d, x}$ and finishes the proof.

So far, by way of classification we have

Proposition 4.4 Let $n \geq 2$ be a positive integer. The left coideal subalgebras of $H_{n^{2}}$ fall into two classes:

- semisimple ones isomorphic to $\mathbb{k}_{\mathrm{k}}^{\prime}$ through Eq. 4.1 for subgroups $G^{\prime} \leq G$, in bijection with right group-like projections; 
- $\quad$ non-semisimple, equal to one of the coideal subalgebras $\mathcal{N}_{d, x}$ in Eq. 4.2 for divisors $d \mid n$.

Proof This is a consequence of Proposition 4.3 together with the fact that a semisimple coideal subalgebra is of the form $\mathcal{N}_{P}$ for a unique right group-like projection $P$ by Proposition 3.23.

Remark 4.5 Note that the non-semisimple coideals do not correspond to group-like projections. Indeed, suppose that $P \in H_{n^{2}}$ is a group-like projection which is an integral for $\mathcal{N}_{d, x}$. Then $P x^{n-1}=0$ and hence $P$ is of the form $y x$ for some $y \in H_{n^{2}}$. But this implies $\varepsilon(P)=0$ - contradiction.

It remains to give a more concrete description of the semisimple coideal subalgebras, forming (as we will see) the bulk of the classification in Proposition 4.4.

Proposition 4.6 Let $n \geq 2$ be an arbitrary positive integer. The semisimple left coideal subalgebras of $H=H_{n^{2}}$ are of one of two types:

- $\quad \mathbb{k} G^{\prime} \subset \mathbb{k} G \subset H$ for some subgroup $G^{\prime} \subset G$;

- $\mathcal{N}_{\beta}:=$ algebra generated by $g+\beta x$ for some $\beta \in \mathbb{k}^{\times}$.

Proof Clearly, all subgroups $G^{\prime}$ give rise to a Hopf subalgebra $\mathbb{k} G^{\prime}$ of $H$ as in the first bullet point above. We thus focus on showing that all other semisimple coideal subalgebras are of the form $\mathcal{N}_{\beta}$ (noting also that all $\mathcal{N}_{\beta}$ are indeed coideal subalgebras, as is easily seen).

Suppose $\mathcal{N}$ is a coideal subalgebra as in the statement. The composition (4.1) identifies $\mathcal{N}$ with some coideal subalgebra of $\mathbb{k} G$, say $\mathbb{k} G^{\prime}$ for a subgroup $G^{\prime} \leq G$ of order $d \mid n$.

Step 1: $\quad G^{\prime}=G$.

For each $s \in G^{\prime}$ the subalgebra $\mathcal{N}$ contains a unique element $s+\psi(s)$ with free term $s$ (i.e. such that $\psi(s)$ has no free term). Our assumption that $\mathcal{N}$ is not $\mathbb{k} G^{\prime}$ means that $\psi: G^{\prime} \rightarrow J(H)$ is not identically zero, and the conclusion we want to reach is that in this case $G^{\prime}=G$ and $\psi(g)=\beta x$ for some scalar $\beta \neq 0$.

Let $s=g^{k d} \in G^{\prime}$ be an element with $y:=\psi(s) \neq 0$. There are two possibilities to consider:

(a): $\operatorname{deg}^{\prime}(y)>1$. Then, applying $\left(\varphi \otimes\right.$ id) $\Delta$ to $y$ for some element $\varphi \in H_{1}^{*}$ will produce a non-zero element in $\mathcal{N} \cap J(H)$, which we are assuming cannot exist. This leaves

(b): $\operatorname{deg}^{\prime}(y)=1$. Suppose $y_{1}$ contains a non-zero scalar multiple of $x g^{j}$ for $j \neq k d-1$. We then have

$$
\Delta\left(y_{1}\right) \sim x g^{j} \otimes g^{j}+g^{j+1} \otimes x g^{j}+\cdots, g^{j+1} \neq g^{k d}
$$

where $\sim$ means 'scalar multiple of' and $\cdots$ signifies the terms resulting from applying $\Delta$ to other monomials $\neq x g^{j}$ appearing in $y_{1}$.

An application of $\varphi \otimes$ id to $\Delta\left(g^{k d}+y\right)$ for $\varphi \in H_{0}^{*}$ dual to $g^{j+1}$ yields a non-zero element $z \in \mathcal{N}$ with no free term (and $z_{1} \in \mathbb{k} x g^{j}$ ). This contradicts the semisimplicity of $\mathcal{N}$, meaning that $y_{1} \in \mathbb{k} x g^{k d-1}$.

Now, however, we have

$$
\Delta\left(y_{1}\right) \sim x g^{k d-1} \otimes g^{k d-1}+g^{k d} \otimes x g^{k d-1},
$$


and setting $\varphi$ dual to $x g^{k d-1},(\varphi \otimes$ id $) \Delta(y)$ will be a non-zero element of $\mathcal{N}$ whose free term is (in the span of) $g^{k d-1}$. Since free terms of elements of $\mathcal{N}$ are in $G^{\prime}=\left\langle g^{d}\right\rangle$, we have $d \mid k d-1$ and hence $d=1$. In conclusion we have $G^{\prime}=G$, as announced earlier.

Step 2: finishing the proof. Now denote $\psi(g)=g+y$. Since we are assuming $\mathcal{N} \neq \mathbb{k}_{k} G$ we have $\operatorname{deg}(y) \geq 1$.

Let $\varphi \in H^{*}$ be the dual element to $g$. The free term of

$$
(\varphi \otimes \mathrm{id}) \circ \Delta(g+y)
$$

is $g$, and since this element belongs to $\mathcal{N}$ it must again be equal to $g+y$. It follows that the degree- $d$-component $y_{d}$ has the property that the $H_{0} \otimes H_{d}$-component of $\Delta(y)$ is in $\mathbb{k} g \otimes H_{d}$, and hence $y_{d} \in \mathbb{k}_{k} x^{d} g^{1-d}$. In other words, we have

$$
y=\psi(g)=\sum_{d>0} \alpha_{d} x^{d} g^{1-d} .
$$

For each term $y_{d}=\alpha_{d} x^{d} g^{1-d}$ the left hand tensorand in the $H_{1} \otimes H_{d-1^{-}}$ component of $\Delta\left(y_{d}\right)$ belongs to the span of $x$, and hence applying $\varphi \otimes$ id to $\Delta(y)$ with $\varphi \in H_{1}^{*}$ dual to $x$ produces a non-zero element of degree $\operatorname{deg}(y)-1$ whose free term is 1 if $\alpha_{1} \neq 0$ and 0 otherwise.

Since $\mathcal{N}$ has no elements with no free terms the second possibility is ruled out. On the other hand, its only element with free term 1 is the unit, so $\operatorname{deg}(y)=1$. This concludes the proof that $y=\beta x$ for some $\beta \in \mathbb{k}^{\times}$.

Corollary 4.7 For $n \geq 2$ the orbits of the coideal subalgebras of $H=H_{n^{2}}$ under the automorphism group of $H$ are as follows:

- $\quad$ a fixed point $\mathbb{k}\left\langle g^{d}\right\rangle$ for each divisor $d \mid n$;

- a fixed point $\mathcal{N}_{d, x}$ for each divisor $d \mid n$;

- the orbit consisting of all $\mathcal{N}_{\beta}$ for $\beta \in \mathbb{k}^{\times}$.

Proof This follows from the classification in Proposition 4.4, the description provided by Proposition 4.6, and the fact that $x \mapsto \beta x$ extends to an automorphism of $H$ for every $\beta \in \mathbb{k}^{\times}$.

The following result is the analogue for right group-like projections. For $\beta \in \mathbb{k}$ we denote by $P_{\beta}$ the idempotent

$$
\frac{1}{n} \sum_{i=0}^{n-1}(g+\beta x)^{i} .
$$

Corollary 4.8 Let $n \geq 2$. The orbits of right group-like projections under the automorphism group of $\mathrm{H}_{n^{2}}$ are the singletons

$$
\left\{\frac{1}{d} \sum_{i=0}^{\frac{n}{d}-1} g^{d i}\right\} \text { for } d \mid n
$$

and $\left\{P_{\beta} \mid \beta \in \mathbb{k}^{\times}\right\}$. 
Note that the right group-like projection $P_{\beta}$ is not preserved by the antipode if $\beta \in \mathbb{k}^{\times}$. This answers the question of M. Landstad and A. Van Daele formulated in [10] where they in particular have shown that it cannot happen in algebraic quantum groups, see [10, Proposition 1.6].

As a separate matter, every automorphism of $H_{n^{2}}$ is of the form $x \mapsto \beta x$ :

Proposition 4.9 Let $n \geq 2$ be an arbitrary positive integer. The map $\beta \mapsto \varphi_{\beta}$ where

$$
\varphi_{\beta}: x \mapsto \beta x, g \mapsto g
$$

is an isomorphism between $\mathbb{k}^{\times}$and the group of automorphisms of $H_{n^{2}}$.

Proof We have already observed that all $\varphi_{\beta}$ are indeed automorphisms; it remains to show that there are no others. To that end, let $\varphi$ be an automorphism of $H=H_{n^{2}}$. Since $H$ is generated by $g$ and $x$, it will be enough to describe the images of these two elements.

Since $G$ is the group of group-like elements, $\varphi$ restricts as an automorphism of $G$. The component

$$
H_{(1)}:=\{x \in H \mid \Delta(x) \in H \otimes \mathbb{k} G+\mathbb{k} G \otimes H\}
$$

of the coradical filtration $[13, \S 5.2]$ of $H$ is

$$
H_{0} \oplus H_{1}=\mathbb{k} G \oplus \operatorname{span}\left\{x g^{i} \mid 0 \leq i \leq n-1\right\} .
$$

For $s, t \in G$ denote

$$
P_{s, t}:=\{x \in H \mid \Delta(x)=x \otimes s+t \otimes x\},
$$

i.e. the space of $(s, t)$-primitive elements $[13, \S 5.4]$. According to [13, Theorem 5.4.1] $H_{(1)}$ is the direct sum of $\mathbb{k}_{k} G$ and all $P_{s, t}^{\prime}$ where the latter are arbitrary spaces satisfying

$$
P_{s, t}=\mathbb{k}(s-t) \oplus P_{s, t}^{\prime} .
$$

We have $x g^{i} \in P_{g^{i}, g^{i+1}}$, so $P_{1, g}=\operatorname{span}\{1-g, x\}$ and $P_{1, s}$ is one-dimensional for all $g \neq s \in G$.

Since $\varphi$ permutes the non-trivial elements $s \in G$ it also permutes the spaces $P_{1, s}$, and hence it must fix the only element (namely $g$ ) whose associated primitive space $P_{1, g}$ is two-dimensional. In conclusion, we have $\varphi(g)=g$.

As for $\varphi(x)$, it must be both an element of $P_{1, g}=\operatorname{span}\{1-g, x\}$ and an $\omega$-eigenvector for conjugation by $g$. The intersection of these two spaces is precisely $\mathbb{k} x$, meaning that $\varphi(x)=\beta x$ for some $\beta \in \mathbb{k}^{\times}$.

Remark 4.10 Let us consider the Taft Hopf algebra over the field of complex numbers. Given $z \in \mathbb{C},|z|=1$ there exists a uniquely determined $*$-structure on $H_{n^{2}}$ such that $g^{*}=g$ and $x^{*}=z x$.

Reasoning as in Proposition 4.9 we can show that there are no other $*$-structures on $H_{n^{2}}$.

Let us equip $H_{n^{2}}$ with the $*$-structure corresponding to $z=1$. Then for $\beta \in \mathbb{R} P_{\beta} \in H_{n^{2}}$ defined (4.3) is self adjoint right group-like projection which is not left; this observation strengthens the answer to the Landstad - Van Daele question.

Remark 4.11 Let us consider the Sweedler's Hopf algebra, $H=H_{4}$. It has a basis $\{\mathbb{1}, g, x, y\}$ where $y=g x, g^{2}=\mathbb{1}, g x g=-x$. Due to Corollary 4.8 the right group-like projections in $H$ are of the form $\mathbb{1}, \frac{1}{2}(\mathbb{1}+g)+\beta x$ where $\beta \in \mathbb{k}$. By direct computations it can be checked that weak group-like projections in $H_{4}$ (see Definition 3.32) are of the form 
$\mathbb{1}$ and $\frac{1}{2}(\mathbb{1}+g)+\beta x+\delta y$ for arbitrary $\beta, \delta \in \mathbb{k}$. In particular the space of classes of weak group-like projections is infinite and can be identified with $\{\mathbb{1}\} \cup\left\{\frac{1}{2}(\mathbb{1}+g)\right\} \cup \mathbb{C P}^{1}$ where $\mathbb{C P}^{1}$ is 1-dimensional complex projective space.

\section{Open Problems}

We formulate a list of questions motivated by the results of the present paper.

(1) Due to the result of [10] there does not exist a right group-like projection in an algebraic quantum group which is not two-sided. Can we prove this result for semisimple or cosemisimple Hopf algebras? Let us note that for cosemisimple Hopf algebras it was recently proved under the assumption that $\mathcal{V}_{P}=\mathcal{N}_{P}$, see [6, Remark 4.8].

(2) Is there a finite dimensional Hopf algebra which has infinitely many classes of left coideal subalgebras?

(3) Is there a finite dimensional Hopf algebra which has infinitely many two sided grouplike projections?

(4) Is there a left coideal subalgebra $\mathcal{N}$ with a two sided counital integral $P$ such that $\mathcal{N}$ is not quasi-Frobenius?

(5) Is there a right group-like projection $P$ such that $\mathcal{N}_{P}$ is not semisimple? Is there a right group-like projection in $\mathcal{A}$ such that $\mathcal{A}$ is not faithfully flat over $\mathcal{N}_{P}$ ?

Acknowledgments The authors are grateful to the anonymous referee for paying our attention to the paper of M. Koppinen, [8]. A.C. was partially supported by NSF grant DMS-1801011. PK was partially supported by the NCN (National Center of Science) grant 2015/17/B/ST1/00085.

Open Access This article is distributed under the terms of the Creative Commons Attribution 4.0 International License (http://creativecommons.org/licenses/by/4.0/), which permits unrestricted use, distribution, and reproduction in any medium, provided you give appropriate credit to the original author(s) and the source, provide a link to the Creative Commons license, and indicate if changes were made.

\section{References}

1. Burciu, S.: Kernels of representations and coideal subalgebras of Hopf algebras. Glasg. Math J. 54(1), 107-119 (2012)

2. Etingof, P., Gelaki, S.: On finite-dimensional semisimple and cosemisimple Hopf algebras in positive characteristic. Internat. Math. Res. Notices 1998(16), 851-864 (1998)

3. Fall, R., Kasprzak, P.: Group-like projections for locally compact quantum groups. J. Operator Theory 80(1), 153-166 (2018)

4. Kalantar, M., Kasprzak, P., Skalski, A.: Open quantum subgroups of locally compact quantum groups. Adv. Math. 303, 322-359 (2016)

5. Kasprzak, P., Sołtan, P.M.: The Lattice of Idempotent States on a Locally Compact Quantum Group. to appear in Publ. RIMS Kyoto Univ.

6. Kasprzak, P.: Generalized (co)integrals on coideal subalgebras. ArXiv e-prints (2018)

7. Kasprzak, P.: Shifts of group-like projections and contractive idempotent functionals for locally compact quantum groups. Internat. J. Math., 29(13) (2018)

8. Koppinen, M.: Coideal subalgebras in Hopf algebras: freeness, integrals, smash products. Comm. Algebra 21(2), 427-444 (1993)

9. Lam, T.Y.: Lectures on modules and rings, vol. 189 of graduate texts in mathematics. Springer, New York (1999)

10. Landstad, M., Van Daele, A.: Compact and discrete subgroups of algebraic quantum groups I. ArXiv Mathematics e-prints (2007) 
11. Larson, R.G., Radford, D.E.: Semisimple cosemisimple Hopf algebras. Amer. J. Math. 110(1), 187-195 (1988)

12. Majid, S.: A Quantum Groups Primer, vol. 292 of London mathematical society lecture note series. Cambridge University Press, Cambridge (2002)

13. Montgomery, S.: Hopf algebras and their actions on rings, vol. 82 of CBMS regional conference series in mathematics. Published for the conference board of the mathematical sciences, Washington, DC; by the American mathematical society, Providence RI (1993)

14. Pevtsova, J., Witherspoon, S.: Varieties for modules of quantum elementary abelian groups. Algebr. Represent. Theory 12(6), 567-595 (2009)

15. Pevtsova, J., Witherspoon, S.: Tensor ideals and varieties for modules of quantum elementary abelian groups. Proc. Amer. Math. Soc. 143(9), 3727-3741 (2015)

16. Radford, D.: Hopf algebras, vol. 49 of series on knots and everything. World Scientific Publishing Co. Pte. Ltd, Hackensack (2012)

17. Skryabin, S.: Projectivity and freeness over comodule algebras. Trans. Amer. Math, Soc. 359(6), 2597$2623(2007)$

18. Skryabin, S.: Finiteness of the number of coideal subalgebras. Proc. Amer. Math Soc. 145(7), 2859-2869 (2017)

19. Taft, E.J.: The order of the antipode of finite-dimensional Hopf algebra. Proc. Nat. Acad. Sci. USA 68, 2631-2633 (1971)

Publisher's Note Springer Nature remains neutral with regard to jurisdictional claims in published maps and institutional affiliations.

\title{
Affiliations
}

\section{Alexandru Chirvasitu ${ }^{1}$ Paweł Kasprzak ${ }^{2}$ (D) . Piotr Szulim ${ }^{3}$}

\author{
Alexandru Chirvasitu \\ achirvas@buffalo.edu \\ Piotr Szulim \\ pw.szulim@student.uw.edu.pl \\ 1 Department of Mathematics, University at Buffalo, Buffalo, NY 14260-2900, USA \\ 2 Department of Mathematical Methods in Physics, Faculty of Physics, University of Warsaw, \\ Warszawa, Poland \\ 3 Faculty of Physics, University of Warsaw, Warszawa, Poland
}

